\title{
Pore network model of primary freeze drying
}

\author{
Vorhauer, N. ${ }^{\text {a*}}$; Först, P. ${ }^{\text {b; Schuchmann, H.c }}$; Tsotsas, E. $^{\text {a }}$

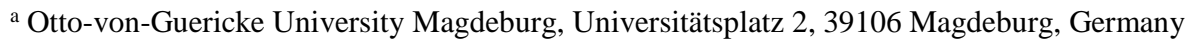 \\ b TU München, Gregor-Mendel-Straße 4, 85354 Freising, Germany \\ ${ }^{\text {c } W i l h e l m ~ B u ̈ c h n e r ~ H o c h s c h u l e, ~ O s t e n d s t r a ß e ~ 3, ~} 64319$ Pfungstadt bei Darmstadt, Germany \\ *E-mail of the corresponding author: nicole.vorhauer@ovgu.de
}

\begin{abstract}
The pore scale progression of the sublimation front during primary freeze drying depends on the local vapor transport and the local heat transfer as well. If the pore space is size distributed, vapor and heat transfer may spatially vary. Beyond that, the pore size distribution can substantially affect the physics of the transport mechanisms if they occur in a transitional regime. Exemplarily, if the critical mean free path is locally exceeded, the vapor transport regime passes from viscous flow to Knudsen diffusion. At the same time, the heat transfer is affected by the local ratio of pore space to the solid skeleton. The impact of the pore size distribution on the transitional vapor and heat transfer can be studied by pore scale models such as the pore network model. As a first approach, we present a pore network model with vapor transport in the transitional regime between Knudsen diffusion and viscous flow at constant temperature in the dry region. We demonstrate the impact of pore size distribution, temperature and pressure on the vapor transport regimes. Then we study on the example of a $2 D$ square lattice, how the presence of micro and macro pores affects the macroscopic progression of the sublimation front.
\end{abstract}

Keywords: pore size distribution; transitional vapor transport; pore network model; fractured sublimation front. 


\section{Introduction}

Primary lyphilization, or freeze drying, is a coupled process of heat and mass transfer through frozen matter, which is usually a block of a frozen solution or a packing of frozen particles, e.g. [1]. The heat of sublimation is usually provided by contact of the frozen material to a shelf with higher temperature and by radiation. The heat transfer from the shelf to the sublimation front, at lower temperature (usually saturation temperature is anticipated at the sublimation front), occurs mainly by heat conduction through the ice phase, if the thermal condutivity of the solid is assumed to be low (usually $\lambda_{\text {ice }} \square \lambda_{\text {solid }}$ ). It is thus plausible that the local distribution of solid and ice phase significantly affects the transport of heat. Especially in case of a strongly heterogeneous distribution of solid and ice, the heat transfer rate can vary locally and affect the structure of the sublimation front. Similarly, vapor transport from the sublimation front to the surface of the frozen material is affected by the distribution of solid and pores, which emerge from sublimation of the ice phase. It is basically expected that more vapor can be transported through larger pores while the occurrence of smaller pores results in the decelaration of drying, e.g. [2]. From this it can be concluded that the drying process might be optimized in terms of drying time and capacity of the dryer based on the control of the distribution of ice crystal sizes (in regard of heat transfer through the ice saturated region of the frozen material) and the distribution of pore sizes (in regard of vapor transfer through already dry regions). For this reason, frozen solutions are foamed to obtain a heterogeneous distribution of pores [3]. Foamed frozen matter is characterized by smaller pores which are saturated with ice at the start of drying and larger unsaturated pores which result from the admixion of gas to the liquid solution before and during the first stage of freezing. As shown in [3] by experiments with a frozen aqueous solution of mannitol stabilized by a mixture of skim milk and sodium carboxymethylcellulose, drying can be much faster using this method, because the transport of vapor is positively affected in presence of the large pores.

It will be discussed in this paper that the simulation of vapor transport through a heterogeneous porous medium of interconnecting and interpenetrating large and small pores, such as in the experiment of [3], represents a special situation in which traditional model approaches or empirical models might rather be substituted by pore network models. The motivation behind that, is the assumption of a transitional vapor transport regime between Knudsen diffusion (in smaller pores) and viscous flow (in larger pores), which cannot be captured by the traditional continuous models or empirical approaches, which essentially blur the different transport properties into effective parameters. Basically, different flow regimes, i.e. either based on a gradient in total pressure (viscous flow) [4] or based on a gradient in vapor pressure (Knudsen diffusion) [5-9] underly the classical models of freeze drying. The distinction between the different flow regimes is generally based on the Knudsen-number, 


$$
K n=\frac{\Lambda}{d}
$$

with pore diameter $d$ and the mean free path of the diffusing molecules

$$
\Lambda=\frac{\mu}{P} \sqrt{\frac{\pi \tilde{R} T}{2}} .
$$

In Eq. (2) $\mu$ denotes the dynamic vapor viscosity in $\mathrm{Pa} \mathrm{s}^{-1}, P$ the pressure in $\mathrm{Pa}, \tilde{R}$ the ideal gas constant in $\mathrm{J} \mathrm{kmol}^{-1} \mathrm{~K}^{-1}$ and $T$ the temperature in $\mathrm{K}$.

Continuum flow (i.e. Darcy flow with no-slip conditions) is expected for $K n<0.001$, while slip flow is expected for $0.001<K n<0.1$. Molecular (Knudsen) flow, based on a concentration gradient of the diffusing substance, occurs for $K n>10$, e.g. [10]. In the transitional range between $K n>0.1$ and $K n<10$, neither the Darcy equation of viscous flow $\left(\dot{m}_{v}=-(\rho k / \mu) \nabla P\right)$ nor the molecular flow equation $\left(\dot{m}_{v}=-D_{K n}(\tilde{M} / \tilde{R} T) \nabla P_{v}\right)$ can provide an exact approach. However, in many situations this range is relevant for freeze drying because of the occurrence of very low pressures, i.e. highly diluted gases. In this range, the approach e.g. presented in [10] can be applied. This is further elaborated in the following section in conjunction with the pore network model.

\section{Pore network model}

The pore network model is based on the concepts e.g. given in [11]. In the simplest case, the porous medium can be represented by a square lattice of pores and throats of varying width and length. Here, we present the results from drying of a pore network with 50x51 pores (4949 throats) of length $L=100 \mu \mathrm{m}$ and with the pore size distribution given in Fig. 1. Note the bimodal distribution of pore sizes, i.e. the presence of pores with smaller radius (peak in Fig. 1) and the tailing of the curve to throats with greater radius (similar as in experiments discussed in [2]). The pores are arranged in a way, that the radii of vertical throats and the pores in every fifth vertical column (i.e. columns $[1,611,16,21,26,31,36$, $41,46,51]$ as indicated by the inlet of the figure) are three times larger than the remaining throats and pores. 


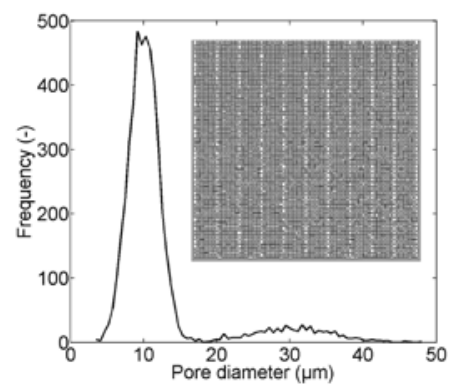

Fig.1 Pore size distribution of the investigated 2D pore network. Note the tailing of the curve towards greater radii (i.e bimdodal pore size distribution).

The simulation is realized with constant temperature $T^{*}=T=-38^{\circ} \mathrm{C}$ and pressure inside the drying chamber $P_{v}^{\infty}=P=10 \mathrm{~Pa}$. The parameters of water are $\tilde{M}_{v}=18.02 \mathrm{~kg} \mathrm{kmol}^{-1}$ (molar mass), ice density $\rho_{\text {ice }}=918 \mathrm{~kg} \mathrm{~m}^{-3}$ and dynamic viscosity of the vapor phase $\mu_{v}=$ $2.5 \cdot 10^{-6} \mathrm{~Pa} \mathrm{~s}^{-1}$. The equilibrium vapor pressure is computed from

$$
\begin{aligned}
& \log _{10} P_{v}^{*}(\text { mbar })=a \cdot\left(T_{0} / T(K)-1\right)+b \cdot \log _{10}\left(T_{0} / T(K)\right)+c \cdot\left(1-T_{0} / T(K)\right) \\
& +\log _{10}\left(P_{v, 0}^{*}\right)
\end{aligned}
$$

with $T_{0}=273.15 \mathrm{~K}, P_{v, 0}^{*}=6.1071$ mbar and $a=-9.09718, b=-3.56654, c=0.876793$. [12]. It is $P_{v}^{*}\left(T^{*}=-38^{\circ} \mathrm{C}\right)=16.0580 \mathrm{~Pa}$.

Computation of vapor transport through the dry zone of the pore network (white throats and pores in Fig. 3 below) follows the semi-empirical approach proposed e.g. in [10] based on the works of Knudsen. The mass flow rate is given by

$$
\dot{M}_{v}\left(k g s^{-1}\right)=-K A \frac{\tilde{M}_{v}}{\tilde{R} T} \frac{\Delta P}{L},
$$

with cross sectional area $A$ in $\mathrm{m}^{2}$, pressure gradient between two neighbor pores $\Delta P$ in $\mathrm{Pa}$ and throat length $L$ in $\mathrm{m}$. The transport coefficient $K$ is computed from

$$
K\left(m^{2} s^{-1}\right)=\frac{d^{2} P}{32 \mu}\left(1+8.88 K n+4.96 K n^{2}\right)
$$

[10] and $\mathrm{Kn}$ from Eq. (1). Each term in Eq. (5) can be related to the contribution of the different flow regimes: the first term of Eq. (5) represents the contribution of Poisseuille flow, the second term of slip flow and the third term of Knudsen flow. Note that the 
calculation of $K$ is an iterative problem if it is assumed that $P=P_{v}$, i.e. the total pressure gradient is equal to the vapor pressure gradient. Then pressure varies with position $x$ (also refer to Fig. 3) and $K$ varies accordingly (Eq. (5)).

Figure 2 shows the dependency of $K$ on pore sizes in the range between $r=1 \mu \mathrm{m}$ and $r$ $=1 \mathrm{~mm}$ with pressure and temperature as in the pore network simulation (Fig. 2a) and for constant pore radii $r=10 \mu \mathrm{m}$ and varying ratio $T / P$ (Fig. 2b). As can be seen, Knudsen flow dominates as long as pore radii are smaller than approximately $10 \mu \mathrm{m}$; however for radii $r>10 \mu \mathrm{m}$ slip flow and Poisseuille flow become increasingly important (Fig. 2a). In the range between $r=1 \mu \mathrm{m}$ and $r=25 \mu \mathrm{m}$ (Fig. 1), $K$ increases almost linearly by about $32 \%$. A similar picture is drawn for constant radius, $r=10 \mu \mathrm{m}$ : In the range $0.01<T / P$ $<100 \mathrm{~K} \mathrm{~Pa}^{-1}$ transitional flow occurs if pressure varies between $\sim 2 \mathrm{~Pa}$ and $\sim 235 \mathrm{hPa}$, leading to a global minimum at $T / P \cong 0.48 \mathrm{~K} \mathrm{~Pa}^{-1}$ (given $T=-38^{\circ} \mathrm{C}$ ).

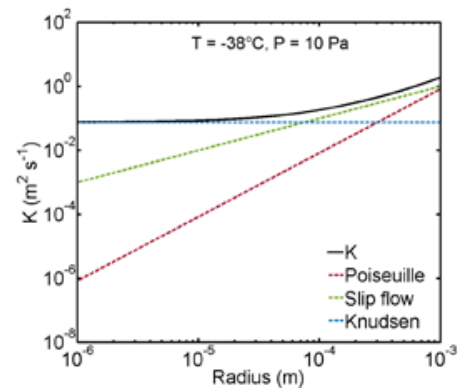

a)

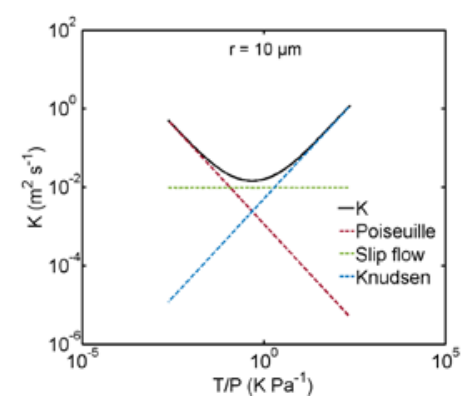

b)

Fig. 2 Contribution of flow regimes to vapor transport a) for constant temperature and pressure and b) for constant radius.

The pore network simulation is started from an initially fully saturated network. The vapor pressure field is computed from the boundary conditions of each pore [11] and the vapor flow rate between two pores is computed from Eq. (4). The sublimation rate from ice saturated pores and throats at the sublimation front is then calculated from

$$
\dot{M}_{\text {sub }}=-\dot{M}_{v}
$$

The vapor pressure field as well as the transport parameters (essentially $K$ ) are updated after the complete emptying of either a throat or a pore. 


\section{Simulation Results}

Figure 3 shows the distribution of ice phase and dry solid phase resulting from a pore network simulation realized with the parameters specified above. Most striking is the structure of the sublimation front evolving in the pore network with bimodal pore size distribution (Fig. 1). Obviously, in the transitional regime vapor flow through larger pores (in every 5th column) is accelerated compared to the flow through smaller pores (in the neighbourhood). This leads to the erosion of the sublimation front and faster progression of the gas phase in pores with greater diameter.

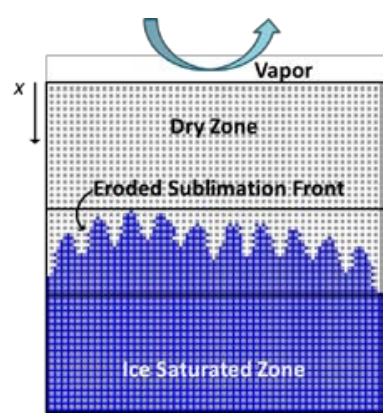

Fig. 3 Freeze drying of a 2D pore network. The ice phase appears in blue, empty pores in white and the solid in gray. Vapor escapes from the top edge.

Figure 4a shows the least advanced position of the sublimation front (measured from the open side of the pore network) as a function of the total ice saturation, i.e. the sublimation front velocity. As can be seen, the macroscopic velocity of the sublimation front is approximately constant in this representation. However, the saturation profiles in Fig. 4b indicate that the front is significantly broadening with progressive invasion of the pore network. This is a result of the assumption of transitional flow in a pore network with bimodal pore structure (Fig. 1) and the higher vapor transport rates in pores with greater radius which allow for higher sublimation rates according to Eq. (6). This is in agreement with experimental results reported in [2]. Furthermore, as indicated by the dashed lines in Fig. 4b, the slice averaged relative humidity, $\varphi=P_{v} / P_{v}^{*}\left(T^{*}\right)$, is approximately $\varphi=1$ in the region of the fractured front and decreases only slightly towards the network surface due to the low spread of vapor pressures $\left(\Delta P=P_{v}^{*}-P_{v}^{\infty}\right.$ ). This shall further be elucidated in future research related to collapse phenomena, e.g. [13]. 


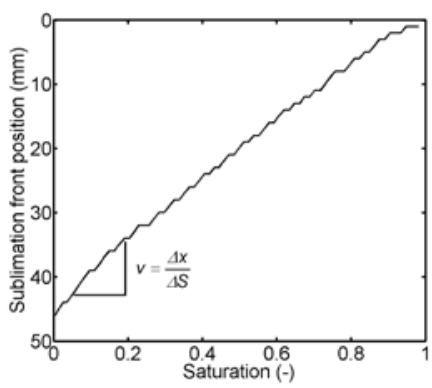

a)

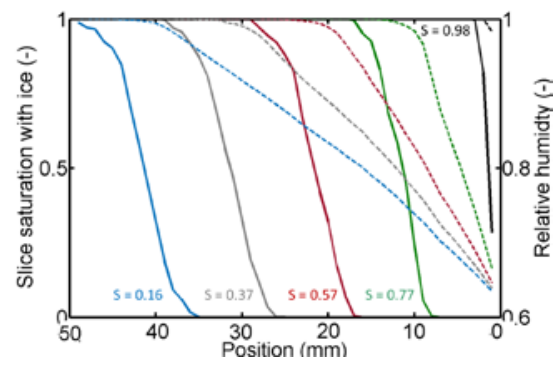

b)

Fig. 4 Analysis of drying kinetics and sublimation front structure: a) sublimation front velocity in terms of $\Delta x / \Delta S, b)$ slice average saturation profiles (solid lines) and relative humidity (dashed lines) for different overall network saturations $S$ with ice phase.

\section{Summary and outlook}

We have presented the results of a pore network simulation of freeze drying applying a bimodal structure of the pore space. It could be shown that in the transitional regime between molecular diffusion and viscous flow, drying can be accelerated due to the presence of macro pores. The interpretation of this outcome is twofold: on the one hand, drying performance can be positively affected by pore design (i.e. in terms of drying time and capacity), on the other hand, higher demands are imposed on process control if the occurance of material collapse is associated with the local drying rate or rather the spatial variation of the velocity of the sublimation front. We thus aim to study in more detail in a future work freeze drying in the transitional regime by 2D and 3D pore network models with different structures of the pore space.

\section{References}

[1] Liapis, A.I.; Bruttini, R. A mathematical model for the spray freeze drying process: The drying of frozen particles in trays and in vials on trays. International Journal of Heat and Mass Transfer 2009, 52, 100-111.

[2] Foerst, P.; Lechner, M.; Vorhauer, N.; Schuchmann, H.; Tsotsas, E. Experimental investigation on pore size distribution and drying kinetics druing lyophilization of sugar solutions. In Proceedings of 21st International Drying Symposium, Valencia, Spain, September 11-14, 2018.

[3] Wang, W.; Hu, D.; Pan, Y.; Zhao, Y.; Chen, G. Freeze-drying of aqueous solution frozen with prebuilt pores. AIChE Journal 2015, 61 (6), 2048-2057. 
[4] Liapis, A.I.; Bruttini, R. Freeze Drying. In: Handbook of Industrial Drying, Mujumdar, A.S., Ed.; CRC Press, Taylor and Francis Group, Boca Raton, 2015, 259-282.

[5] Hottot, A.; Vessot, S.; Andrieu, J. A direct characterization method of the ice morphology. Relationship between mean crystals size and primary drying times of freeze drying processes. Drying Technology 2004, 22 (8), 2009-2021.

[6] Rasetto, V.; Marchisio, D.L.; Fissore, D.; Barresi, A.A. On the use of a dual-scale model to improve understanding of a pharmaceutical freeze-drying process. Journal of Pharmaceutical Sciences 2010, 99 (10), 4337-4350.

[7] Raman, P.; Rielly, C.D.; Stapley, A.G.F. Freeze drying microscopy as a tool to study the sublimation kinetics of a freeze drying process. In: In Proceedings of 19th International Drying Symposium, Lyon, France, August 24-27, 2014.

[8] Chitu, T.; Vessot, S.; Peczalsky, R.; Andrieu, J.; Woinet, B.; Francon, A. Influence of operating conditions on the freeze-drying of frozen particles in a fixed bed and modeling data. Drying Technology 2015, 3, 1892-1898.

[9] Trelea, I.C.; Passot, S.; Marin, M.; Fonseca, F. Model for heat and mass transfer in freeze drying of pellets. Journal of Biomechanical Engineering 2009, 131, 074501-1.

[10] Zarekar, S.; Bück, A.; Jacob, M.; Tsotsas, E. Reconsideration of the hydrodynamic behavior of fluidized beds operated under reduced pressure. Powder Technology 2016, 287, 169-176.

[11] Metzger, T.; Tsotsas, E.; Prat, M. Pore-network models: A powerful tool to study drying at the pore level and understand the influence of structure on drying kinetics. In: Modern Drying Technology, Vol. 1: Computational Tools at Different Scales; Tsotsas, E., Mujumdar A.S., Eds.; Wiley-VCH: Weinheim, 2007, 57-102.

[12] Goff, J.A.; Gratch, S. Low-pressure properties of water from $-160^{\circ}$ to $212^{\circ} \mathrm{F}$. Trans. Amer. Soc. Heat Vent. Eng. 1946, 52, 95-121.

[13] Meister, E.; Gieseler, H. Freeze-dry microscopy of protein/sugar mixtures: Drying behavior, interpretation of collapse temperatures and a comparison to corresponding glass transition data. J. Pharm. Sci. 2008, 98, 3072-3087. 